\title{
Global and Blow-Up Solutions for a Class of Nonlinear Parabolic Problems under Robin Boundary Condition
}

\author{
Lingling Zhang and Hui Wang \\ Department of Mathematics, Taiyuan University of Technology, Shanxi 030024, China \\ Correspondence should be addressed to Lingling Zhang; zhangllwangh@126.com
}

Received 26 June 2014; Accepted 28 July 2014; Published 14 August 2014

Academic Editor: Sanling Yuan

Copyright ( 2014 L. Zhang and H. Wang. This is an open access article distributed under the Creative Commons Attribution License, which permits unrestricted use, distribution, and reproduction in any medium, provided the original work is properly cited.

We discuss the global and blow-up solutions of the following nonlinear parabolic problems with a gradient term under Robin boundary conditions: $(b(u))_{t}=\nabla \cdot(h(t) k(x) a(u) \nabla u)+f\left(x, u,|\nabla u|^{2}, t\right)$, in $D \times(0, T),(\partial u / \partial n)+\gamma u=0$, on $\partial D \times(0, T), u(x, 0)=$ $u_{0}(x)>0$, in $\bar{D}$, where $D \subset \mathbb{R}^{N}(N \geq 2)$ is a bounded domain with smooth boundary $\partial D$. Under some appropriate assumption on the functions $f, h, k, b$, and $a$ and initial value $u_{0}$, we obtain the sufficient conditions for the existence of a global solution, an upper estimate of the global solution, the sufficient conditions for the existence of a blow-up solution, an upper bound for "blow-up time," and an upper estimate of "blow-up rate." Our approach depends heavily on the maximum principles.

\section{Introduction}

The study of global and blow-up solutions for nonlinear parabolic equations has received a lot of attention in the past several decades (see [1-4]). In most works, so far, a variety of approaches have been developed in dealing with different nonlinear parabolic problems, such as the existence of global solution, blow-up solution, an upper bound for "blow-up time," an upper estimate of "blow-up rate," or global solution. So far, some applications in physics, chemistry, and biology are relevant to blow-up phenomena which can be found in [5-11]. In this paper, we consider the global and blow-up solutions of the following nonlinear parabolic equation with Robin boundary condition:

$$
\begin{array}{r}
(b(u))_{t}=\nabla \cdot(h(t) k(x) a(u) \nabla u)+f(x, u, q, t), \\
\text { in } D \times(0, T), \\
\frac{\partial u}{\partial n}+\gamma u=0, \quad \text { on } \partial D \times(0, T), \\
u(x, 0)=u_{0}(x)>0, \quad \text { in } \bar{D},
\end{array}
$$

where $q=|\nabla u|^{2}, D \subset \mathbb{R}^{N}(N \geq 2)$ is a bounded domain with smooth boundary $\partial D, \partial u / \partial n$ represents the outward normal derivative on $\partial D, \gamma$ is positive constant, $u_{0}$ is the initial value,
$T$ is the maximal existence time of $u$, and $\bar{D}$ is the closure of $D$. Set $\mathbb{R}^{+}=(0,+\infty)$. We assume, throughout the paper, that $b(s)$ is a positive $C^{3}\left(\mathbb{R}^{+}\right)$function, $b^{\prime}(s)>0$ for any $s \in \mathbb{R}^{+}$, $a(s)$ is a positive $C^{2}\left(\mathbb{R}^{+}\right)$function, $k(x)$ is a positive $C^{1}(\bar{D})$ function, $h(t)$ is a positive $C^{1}\left(\mathbb{R}^{+}\right)$function, $f(x, s, d, t)$ is a nonnegative $C^{1}\left(\bar{D} \times \mathbb{R}^{+} \times \overline{\mathbb{R}^{+}} \times \mathbb{R}^{+}\right)$function, and $u_{0}(x)$ is a positive $C^{2}(\bar{D})$ function. Under these assumptions, the classical parabolic equation theory [12] ensures that there exists a unique classical solution $u(x, t)$ with some $T>0$ for the problem (1), and the solution is positive over $\bar{D} \times[0, T)$. Moreover, by the regularity theorem [13], $u(x, t) \in C^{3}(D \times$ $(0, T)) \cap C^{2}(\bar{D} \times[0, T))$.

The problems of the global and blow-up solutions for nonlinear parabolic equations have been investigated extensively by many authors and have got a lot of meaningful results. Some special cases of problem (1) have been treated already. Ding [14] deals with the following problem:

$$
\begin{gathered}
(b(u))_{t}=\nabla \cdot(a(u) \nabla u)+f(u), \quad \text { in } D \times(0, T), \\
\frac{\partial u}{\partial n}+\gamma u=0, \quad \text { on } \partial D \times(0, T), \\
u(x, 0)=h(x)>0, \quad \text { in } \bar{D},
\end{gathered}
$$


where $D$ is a bounded domain of $\mathbb{R}^{N}(N \geq 2)$ with smooth boundary $\partial D$. By constructing auxiliary functions and using a first-order differential inequality technique, Ding derives conditions on the data, which guarantee the existence of blow-up or global solution. The following problem is investigated by Enache in [15]:

$$
\begin{gathered}
u_{t}=\nabla \cdot(a(u) \nabla u)+f(u), \quad \text { in } D \times(0, T), \\
\frac{\partial u}{\partial n}+\gamma u=0, \quad \text { on } \partial D \times(0, T), \\
u(x, 0)=h(x)>0, \quad \text { in } \bar{D},
\end{gathered}
$$

where $D$ is a bounded domain of $\mathbb{R}^{N}(N \geq 2)$ with smooth boundary $\partial D$. By constructing auxiliary functions and firstorder differential inequality technique, Enache establishes some conditions on nonlinearities and the initial date to guarantee that $u(x, t)$ exists for all times $t>0$ or blows up at some finite time $T$. Besides, the following problem is investigated by Zhang in [16]:

$$
\begin{gathered}
(b(u))_{t}=\Delta u+f(u), \quad \text { in } D \times(0, T), \\
\frac{\partial u}{\partial n}+\gamma u=0, \quad \text { on } \partial D \times(0, T), \\
u(x, 0)=h(x)>0, \quad \text { in } \bar{D}
\end{gathered}
$$

where $D$ is a bounded domain in $\mathbb{R}^{N}(N \geq 2)$ with smooth boundary. Under appropriate assumptions on the functions $f, b$, and $h$, Zhang obtains the conditions under which the solutions may exist globally or blow up in a finite time. Moreover, upper estimates of the "blow-up time," blow-up rate, and global solutions are obtained also.

In this paper, we obtain the existence theorem of global and blow-up solution by constructing completely different auxiliary functions and technically using maximum principles. As a result, the sufficient conditions for the existence of a global solution and an upper estimate of the global solution and the sufficient conditions for the existence of a blow-up solution, an upper bound for "blow-up time," and an upper estimate of "blow-up rate" are specified under some appropriate assumption on the functions $f, h, k, b$, and $a$ and initial value $u_{0}$. Our results extend and supplement those obtained in [14-16].

The content of this paper is organized as follows. In Section 2, we study the existence of the global solution of (1). In Section 3, we investigate the blow-up solution of (1). In Section 4, we will give a few examples to explain our results.

\section{Global Solution}

Our main result for the global solution is the following Theorem 1.

Theorem 1. Let $u$ be a solution of (1). Suppose that the following conditions (i)-(iv) are satisfied. (i) For any $s \in \mathbb{R}^{+}$,

$$
\begin{gathered}
\left(s b^{\prime}(s)\right)^{\prime} \geq 0, \\
s b^{\prime}(s)-\left(s b^{\prime}(s)\right)^{\prime} \leq 0 \\
\left(\frac{a(s)}{b^{\prime}(s)}\right)^{\prime} \leq 0 \\
{\left[\frac{1}{a(s)}\left(\frac{a(s)}{b^{\prime}(s)}\right)^{\prime}+\frac{1}{b^{\prime}(s)}\right]^{\prime}+\frac{1}{a(s)}\left(\frac{a(s)}{b^{\prime}(s)}\right)^{\prime}+\frac{1}{b^{\prime}(s)} \leq 0 .}
\end{gathered}
$$

(ii) For any $(x, s, d, t) \in D \times \mathbb{R}^{+} \times \overline{\mathbb{R}^{+}} \times \mathbb{R}^{+}$,

$$
\begin{gathered}
\left(\frac{f(x, s, d, t)}{h(t)}\right)_{t} \leq 0, \\
f_{d}(x, s, d, t)\left[\left(\frac{1}{b^{\prime}(s)}\right)^{\prime}+\frac{1}{b^{\prime}(s)}\right] \leq 0, \\
\left(\frac{f(x, s, d, t) b^{\prime}(s)}{a(s)}\right)_{s}-\frac{f(x, s, d, t) b^{\prime}(s)}{a(s)} \\
+\frac{h^{\prime}(t)\left(b^{\prime}(s)\right)^{2}}{a(s) h(t)} \leq 0 .
\end{gathered}
$$

(iii) Consider the integration

$$
\int_{m_{0}}^{+\infty} \frac{b^{\prime}(s)}{e^{s}} d s=+\infty, \quad m_{0}=\min _{\bar{D}} u_{0}(x) .
$$

(iv) Consider

$$
\begin{array}{r}
\alpha=\max _{\bar{D}}\left\{\frac{\nabla \cdot\left(h(0) k(x) a\left(u_{0}\right) \nabla u_{0}\right)+f\left(x, u_{0}, q_{0}, 0\right)}{e^{u_{0}}}\right\}>0, \\
q_{0}=\left|\nabla u_{0}\right|^{2} .
\end{array}
$$
and

Then the solution $u$ to problem (1) must be a global solution

$$
u(x, t) \leq H^{-1}\left(\alpha t+H\left(u_{0}(x, t)\right)\right), \quad(x, t) \in \bar{D} \times \overline{\mathbb{R}^{+}},
$$

where

$$
H(z)=\int_{m_{0}}^{z} \frac{b^{\prime}(s)}{e^{s}} d s, \quad z \geq m_{0},
$$

and $H^{-1}$ is the inverse function of $H$.

Proof. Consider the auxiliary function

$$
P(x, t)=b^{\prime}(u) u_{t}-\alpha e^{u}
$$


Then, we have

$$
\begin{gathered}
\nabla P=b^{\prime \prime} u_{t} \nabla u+b^{\prime} \nabla u_{t}-\alpha e^{u} \nabla u, \\
\Delta P=b^{\prime \prime \prime} u_{t}|\nabla u|^{2}+2 b^{\prime \prime} \nabla u \cdot \nabla u_{t} \\
+b^{\prime \prime} u_{t} \Delta u+b^{\prime} \Delta u_{t}-\alpha e^{u}|\nabla u|^{2}-\alpha e^{u} \Delta u .
\end{gathered}
$$

By (1),

$$
\begin{aligned}
(b(u))_{t}= & b^{\prime} u_{t}=\nabla \cdot(h(t) k(x) a(u) \nabla u)+f \\
= & h(t) k(x) a(u) \Delta u+h(t) k(x) a^{\prime}(u)|\nabla u|^{2} \\
& \quad+h(t) a(u)(\nabla k \cdot \nabla u)+f .
\end{aligned}
$$

We have

$$
\begin{aligned}
u_{t}= & \frac{a k h}{b^{\prime}} \Delta u+\frac{a^{\prime} k h}{b^{\prime}}|\nabla u|^{2}+\frac{a h}{b^{\prime}}(\nabla k \cdot \nabla u)+\frac{f}{b^{\prime}} \\
\left(u_{t}\right)_{t}= & h^{\prime}\left(\frac{a k}{b^{\prime}} \Delta u+\frac{a^{\prime} k}{b^{\prime}}|\nabla u|^{2}+\frac{a}{b^{\prime}}(\nabla k \cdot \nabla u)\right) \\
& +h\left(\frac{a k}{b^{\prime}} \Delta u+\frac{a^{\prime} k}{b^{\prime}}|\nabla u|^{2}+\frac{a}{b^{\prime}}(\nabla k \cdot \nabla u)\right)_{t} \\
& +\left(\frac{f}{b^{\prime}}\right)_{t} \\
= & \left.\frac{a^{\prime \prime}}{b^{\prime}}-\frac{a^{\prime} b^{\prime \prime}}{\left(b^{\prime}\right)^{2}}\right) h k u_{t}|\nabla u|^{2}+\frac{2 a^{\prime} k h}{b^{\prime}}\left(\nabla u \cdot \nabla u_{t}\right) \\
& +\left(\frac{a^{\prime}}{b^{\prime}}-\frac{a b^{\prime \prime}}{\left(b^{\prime}\right)^{2}}\right) h u_{t}(\nabla k \cdot \nabla u) \\
& +\frac{f_{u} u_{t}+2 f_{q}\left(\nabla u \cdot \nabla u_{t}\right)+f_{t}}{b^{\prime}}-\frac{f b^{\prime \prime} u_{t}}{\left(b^{\prime}\right)^{2}} \\
& \left.+\frac{a k h}{b^{\prime}} \Delta u_{t}+\frac{a k h^{\prime}}{b^{\prime}} \Delta u \cdot \nabla u_{t}\right)+\left.\left(\frac{a^{\prime}}{b^{\prime}}-\frac{a b^{\prime \prime}}{\left(b^{\prime}\right)^{2}}\right) h k u_{t} \Delta u\right|^{2}+\frac{a h^{\prime}}{b^{\prime}}(\nabla k \cdot \nabla u)
\end{aligned}
$$

Then

$$
\begin{aligned}
P_{t}= & b^{\prime \prime}\left(u_{t}\right)^{2}+b^{\prime}\left(u_{t}\right)_{t}-\alpha e^{u} u_{t} \\
= & b^{\prime \prime}\left(u_{t}\right)^{2}+\left(a^{\prime}-\frac{a b^{\prime \prime}}{b^{\prime}}\right) k h u_{t} \Delta u+a k h \Delta u_{t} \\
& +a k h^{\prime} \Delta u+\left(a^{\prime \prime}-\frac{a^{\prime} b^{\prime \prime}}{b^{\prime}}\right) k h u_{t}|\nabla u|^{2}+a^{\prime} k h^{\prime}|\nabla u|^{2} \\
& +\left(2 a^{\prime} k h+2 f_{q}\right)\left(\nabla u \cdot \nabla u_{t}\right)
\end{aligned}
$$

$$
\begin{aligned}
& +\left(a^{\prime}-\frac{a b^{\prime \prime}}{b^{\prime}}\right) h u_{t}(\nabla k \cdot \nabla u)+a h\left(\nabla k \cdot \nabla u_{t}\right) \\
& +a h^{\prime}(\nabla k \cdot \nabla u) \\
& +\left(f_{u}-\frac{f b^{\prime \prime}}{b^{\prime}}-\alpha e^{u}\right) u_{t}+f_{t} .
\end{aligned}
$$

By (13) and (16), it follows that

$$
\begin{aligned}
\frac{a k h}{b^{\prime}} \Delta & P-P_{t} \\
= & \left(\frac{a k h b^{\prime \prime \prime}}{b^{\prime}}+\frac{a^{\prime} k h b^{\prime \prime}}{b^{\prime}}-a^{\prime \prime} k h\right) u_{t}|\nabla u|^{2} \\
& +\left(\frac{2 a k h b^{\prime \prime}}{b^{\prime}}-2 a^{\prime} k h-2 f_{q}\right)\left(\nabla u \cdot \nabla u_{t}\right) \\
& +\left(\frac{2 a k h b^{\prime \prime}}{b^{\prime}}-a^{\prime} k h\right) u_{t} \Delta u \\
& -\left(\frac{a k h}{b^{\prime}} \alpha e^{u}+a^{\prime} h^{\prime} k\right)|\nabla u|^{2}-\left(\frac{a k h}{b^{\prime}} \alpha e^{u}+a k h^{\prime}\right) \Delta u \\
& -b^{\prime \prime}\left(u_{t}\right)^{2}+\left(\frac{f b^{\prime \prime}}{b^{\prime}}+\alpha e^{u}-f_{u}\right) u_{t} \\
& +\left(\frac{a h b^{\prime \prime}}{b^{\prime}}-a^{\prime} h\right) u_{t}(\nabla k \cdot \nabla u) \\
& -a h\left(\nabla k \cdot \nabla u_{t}\right)-a h^{\prime}(\nabla k \cdot \nabla u)-f_{t} .
\end{aligned}
$$

By (14), we have

$$
\Delta u=\frac{b^{\prime}}{a k h} u_{t}-\frac{a^{\prime}}{a}|\nabla u|^{2}-\frac{1}{k}(\nabla k \cdot \nabla u)-\frac{f}{a k h} .
$$

Substitute (18) into (17) to get

$$
\begin{aligned}
\frac{a k h}{b^{\prime}} \Delta & P-P_{t} \\
= & \left(\frac{a k h b^{\prime \prime \prime}}{b^{\prime}}+\frac{\left(a^{\prime}\right)^{2} k h}{a}-\frac{a^{\prime} k h b^{\prime \prime}}{b^{\prime}}-a^{\prime \prime} k h\right) u_{t}|\nabla u|^{2} \\
& +\left(\frac{2 a k h b^{\prime \prime}}{b^{\prime}}-2 a^{\prime} k h-2 f_{q}\right)\left(\nabla u \cdot \nabla u_{t}\right) \\
& +\left(b^{\prime \prime}-\frac{a^{\prime} b^{\prime}}{a}\right) u_{t}^{2}+\left(\frac{a^{\prime} f}{a}-\frac{f b^{\prime \prime}}{b^{\prime}}-f_{u}-\frac{b^{\prime} h^{\prime}}{h}\right) u_{t} \\
& +\left(\frac{a^{\prime} k h}{b^{\prime}} \alpha e^{u}-\frac{a k h}{b^{\prime}} \alpha e^{u}\right)|\nabla u|^{2}
\end{aligned}
$$




$$
\begin{aligned}
& -\frac{a h b^{\prime \prime}}{b^{\prime}} u_{t}(\nabla k \cdot \nabla u)-a h\left(\nabla k \cdot \nabla u_{t}\right) \\
& +\frac{a h}{b^{\prime}} \alpha e^{u}(\nabla k \cdot \nabla u)+\frac{f}{b^{\prime}} \alpha e^{u}+\frac{f h^{\prime}}{h}-f_{t} .
\end{aligned}
$$

By (12), we have

$$
\nabla u_{t}=\frac{1}{b^{\prime}} \nabla P-\frac{b^{\prime \prime}}{b^{\prime}} u_{t} \nabla u+\alpha \frac{e^{u}}{b^{\prime}} \nabla u .
$$

Next, we substitute (20) into (19) to obtain

$$
\begin{aligned}
\frac{a k h}{b^{\prime}} \Delta & P-P_{t} \\
= & \left(\frac{2 a k h b^{\prime \prime}}{\left(b^{\prime}\right)^{2}}-\frac{2 a^{\prime} k h}{b^{\prime}}-\frac{2 f_{q}}{b^{\prime}}\right)(\nabla u \cdot \nabla P)-\frac{a h}{b^{\prime}}(\nabla k \cdot \nabla P) \\
+ & \left(\frac{a k h b^{\prime \prime \prime}}{b^{\prime}}-\frac{2 a k h\left(b^{\prime \prime}\right)^{2}}{\left(b^{\prime}\right)^{2}}+\frac{a^{\prime} k h b^{\prime \prime}}{b^{\prime}}-a^{\prime \prime} k h\right. \\
+ & \left.+\frac{\left(a^{\prime}\right)^{2} k h}{a}+\frac{2 b^{\prime \prime} f_{q}}{b^{\prime}}\right) u_{t}|\nabla u|^{2} \\
+ & \left(b^{\prime \prime}-\frac{a^{\prime} b^{\prime}}{a}\right) u_{t}^{2}+\left(\frac{a^{\prime} f}{a}-\frac{f b^{\prime \prime}}{b^{\prime}}-f_{u}-\frac{b^{\prime} h^{\prime}}{h}\right) u_{t} \\
+ & \frac{f}{b^{\prime}} \alpha e^{u}+\frac{f h^{\prime}}{h}-f_{t} .
\end{aligned}
$$

So we have

$$
\begin{aligned}
\frac{a k h}{b^{\prime}} \Delta P+\left(\frac{2 a^{\prime} k h}{b^{\prime}}+\frac{2 f_{q}}{b^{\prime}}-\frac{2 a k h b^{\prime \prime}}{\left(b^{\prime}\right)^{2}}\right) \\
\times(\nabla u \cdot \nabla P)+\frac{a h}{b^{\prime}}(\nabla k \cdot \nabla P)-P_{t} \\
=\left(\frac{a k h b^{\prime \prime \prime}}{b^{\prime}}-\frac{2 a k h\left(b^{\prime \prime}\right)^{2}}{\left(b^{\prime}\right)^{2}}+\frac{a^{\prime} k h b^{\prime \prime}}{b^{\prime}}-a^{\prime \prime} k h+\frac{\left(a^{\prime}\right)^{2} k h}{a}\right. \\
\left.+\frac{2 b^{\prime \prime} f_{q}}{b^{\prime}}\right) u_{t}|\nabla u|^{2} \\
+\left(\frac{2 a k h b^{\prime \prime}}{\left(b^{\prime}\right)^{2}} \alpha e^{u}-\frac{a^{\prime} k h}{b^{\prime}} \alpha e^{u}-\frac{a k h}{b^{\prime}} \alpha e^{u}-\frac{2 f_{q}}{b^{\prime}} \alpha e^{u}\right)|\nabla u|^{2} \\
+\left(b^{\prime \prime}-\frac{a^{\prime} b^{\prime}}{a}\right) u_{t}^{2}+\left(\frac{a^{\prime} f}{a}-\frac{f b^{\prime \prime}}{b^{\prime}}-f_{u}-\frac{b^{\prime} h^{\prime}}{h}\right) u_{t} \\
+\frac{f}{b^{\prime}} \alpha e^{u}+\frac{f h^{\prime}}{h}-f_{t} .
\end{aligned}
$$

According to (11), we have

$$
u_{t}=\frac{1}{b^{\prime}} P+\alpha \frac{e^{u}}{b^{\prime}}
$$

Substituting (23) into (22), we have

$$
\begin{aligned}
\frac{a k h}{b^{\prime}} \Delta P+\left[2 k h\left(\frac{a}{b^{\prime}}\right)^{\prime}+\frac{2 f_{q}}{b^{\prime}}\right](\nabla u \cdot \nabla P)+\frac{a h}{b^{\prime}}(\nabla k \cdot \nabla P) \\
+\left[\frac{a}{\left(b^{\prime}\right)^{2}}\left(\frac{f b^{\prime}}{a}\right)_{u}+\frac{h^{\prime}}{h}\right] P \\
+\left[a k h\left(\frac{1}{a}\left(\frac{a}{b^{\prime}}\right)^{\prime}\right)^{\prime}+2 f_{q}\left(\frac{1}{b^{\prime}}\right)^{\prime}\right]|\nabla u|^{2} P-P_{t} \\
=\left(-\alpha e^{u}\right)\left(\frac{2 f_{q}}{b^{\prime}}-\frac{2 b^{\prime \prime} f_{q}}{\left(b^{\prime}\right)^{2}}\right)|\nabla u|^{2} \\
+\left(-\alpha e^{u}\right) k h\left(\frac{a^{\prime}}{b^{\prime}}-\frac{a b^{\prime \prime}}{\left(b^{\prime}\right)^{2}}\right)|\nabla u|^{2} \\
+\alpha e^{u}\left(\frac{a^{\prime} f}{a b^{\prime}}-\frac{f b^{\prime \prime}}{\left(b^{\prime}\right)^{2}}-\frac{f_{u}}{b^{\prime}}+\frac{f}{b^{\prime}}-\frac{h^{\prime}}{h}\right)+\frac{h^{\prime}}{h} f-f_{t} . \\
+\alpha k h b^{\prime \prime \prime} \\
+\frac{\left(b^{\prime}\right)^{2}}{2 a k h\left(b^{\prime \prime}\right)^{2}} \\
\left.+\frac{\left(a^{\prime}\right)^{2} k h}{a b^{\prime}}\right)^{3}|\nabla u|^{2} \\
+\frac{a^{\prime} k h b^{\prime \prime}}{\left(b^{\prime}\right)^{2}}-\frac{a^{\prime \prime} k h}{b^{\prime}}
\end{aligned}
$$

Namely,

$$
\begin{gathered}
\frac{a k h}{b^{\prime}} \Delta P+\left[\left(2 k h\left(\frac{a}{b^{\prime}}\right)^{\prime}+\frac{2 f_{q}}{b^{\prime}}\right) \nabla u+\frac{a h}{b^{\prime}} \nabla k\right] \cdot \nabla P \\
+\left\{\frac{a}{\left(b^{\prime}\right)^{2}}\left(\frac{f b^{\prime}}{a}\right)_{u}+\frac{h^{\prime}}{h}\right. \\
\left.+\left[a k h\left(\frac{1}{a}\left(\frac{a}{b^{\prime}}\right)^{\prime}\right)^{\prime}+2 f_{q}\left(\frac{1}{b^{\prime}}\right)^{\prime}\right]|\nabla u|^{2}\right\} P-P_{t} \\
=-\alpha e^{u}\left\{2 f_{q}\left[\left(\frac{1}{b^{\prime}}\right)^{\prime}+\frac{1}{b^{\prime}}\right]+a k h\right. \\
\left.\times\left[\left(\frac{1}{a}\left(\frac{a}{b^{\prime}}\right)^{\prime}+\frac{1}{b^{\prime}}\right)^{\prime}+\frac{1}{a}\left(\frac{a}{b^{\prime}}\right)^{\prime}+\frac{1}{b^{\prime}}\right]\right\}|\nabla u|^{2}
\end{gathered}
$$




$$
\begin{aligned}
& -\frac{\left(b^{\prime}\right)^{2}}{a}\left(\frac{a}{b^{\prime}}\right)^{\prime} u_{t}^{2}-\alpha e^{u} \frac{a}{\left(b^{\prime}\right)^{2}} \\
& \times\left[\left(\frac{f b^{\prime}}{a}\right)_{u}-\frac{f b^{\prime}}{a}+\frac{h^{\prime}\left(b^{\prime}\right)^{2}}{a h}\right]-h\left(\frac{f}{h}\right)_{t}
\end{aligned}
$$

The assumptions (5) and (6) guarantee that the right-hand side of (25) is nonnegative; that is,

$$
\begin{aligned}
\frac{a k h}{b^{\prime}} \Delta P+ & {\left[\left(2 k h\left(\frac{a}{b^{\prime}}\right)^{\prime}+\frac{2 f_{q}}{b^{\prime}}\right) \nabla u+\frac{a h}{b^{\prime}} \nabla k\right] \cdot \nabla P } \\
+ & \left\{\frac{a}{\left(b^{\prime}\right)^{2}}\left(\frac{f b^{\prime}}{a}\right)_{u}+\frac{h^{\prime}}{h}\right. \\
+ & {\left.\left[a k h\left(\frac{1}{a}\left(\frac{a}{b^{\prime}}\right)^{\prime}\right)^{\prime}+2 f_{q}\left(\frac{1}{b^{\prime}}\right)^{\prime}\right]|\nabla u|^{2}\right\} P-P_{t} }
\end{aligned}
$$

$\geq 0, \quad$ in $D \times(0, T)$.

By applying maximum principle (see [17]), it follows from (26) that $P$ can attain its nonnegative maximum only for $\bar{D} \times\{0\}$ or $\partial D \times(0, T)$.

For $\bar{D} \times\{0\}$, by (8), we have

$$
\begin{aligned}
& \max _{\bar{D}} P(x, 0) \\
& =\max _{\bar{D}}\left\{b^{\prime}\left(u_{0}\right)\left(u_{0}\right)_{t}-\alpha e^{u_{0}}\right\} \\
& =\max _{\bar{D}}\left\{\left[\nabla \cdot\left(h(0) k(x) a\left(u_{0}\right) \nabla u_{0}\right)\right.\right. \\
& \left.\left.\quad+f\left(x, u_{0}, q_{0}, 0\right)\right]-\alpha e^{u_{0}}\right\} \\
& =\max _{\bar{D}}\left\{e ^ { u _ { 0 } } \left[\frac{\nabla \cdot\left(h(0) k(x) a\left(u_{0}\right) \nabla u_{0}\right)+f\left(x, u_{0}, q_{0}, 0\right)}{e^{u_{0}}}\right.\right. \\
& -\alpha]\}=0 .
\end{aligned}
$$

For $\partial D \times(0, T)$, we claim that $P$ cannot take a positive maximum at any point $(x, t)$. In fact, suppose that $P$ can take a positive maximum at one point $\left(x_{0}, t_{0}\right) \in \partial D \times(0, T)$; then

$$
P\left(x_{0}, t_{0}\right)>0,\left.\quad \frac{\partial P}{\partial n}\right|_{\left(x_{0}, t_{0}\right)}>0 .
$$

Combine (1) and (11) with (23); we have

$$
\begin{aligned}
\frac{\partial P}{\partial n} & =b^{\prime \prime} u_{t} \frac{\partial u}{\partial n}+b^{\prime} \frac{\partial u_{t}}{\partial n}-\alpha e^{u} \frac{\partial u}{\partial n} \\
& =-\gamma b^{\prime \prime} u u_{t}+b^{\prime}\left(\frac{\partial u}{\partial n}\right)_{t}+\gamma \alpha u e^{u} \\
& =-\gamma b^{\prime \prime} u u_{t}+b^{\prime}(-\gamma u)_{t}+\gamma \alpha u e^{u}
\end{aligned}
$$

$$
\begin{aligned}
& =-\gamma\left(u b^{\prime}\right)^{\prime} u_{t}+\gamma \alpha u e^{u} \\
& =-\gamma\left(u b^{\prime}\right)^{\prime}\left(\frac{1}{b^{\prime}} P+\alpha \frac{1}{b^{\prime}} e^{u}\right)+\gamma \alpha u e^{u} \\
& =-\gamma \frac{\left(u b^{\prime}\right)^{\prime}}{b^{\prime}} P+\gamma \alpha e^{u} \frac{u b^{\prime}-\left(u b^{\prime}\right)^{\prime}}{b^{\prime}}, \\
& \text { on } \partial D \times(0, T) .
\end{aligned}
$$

Next, by using a part condition of $(5)\left(s b^{\prime}(s)\right)^{\prime} \geq 0, s b^{\prime}(s)-$ $\left(s b^{\prime}(s)\right)^{\prime} \leq 0$ for any $s \in \mathbb{R}^{+}$, we can obtain

$$
\left.\frac{\partial P}{\partial n}\right|_{\left(x_{0}, t_{0}\right)} \leq 0,
$$

which contradicts with inequality (28). Thus, we know that the maximum of $P$ in $\bar{D} \times[0, T)$ is zero; that is,

$$
P \leq 0, \quad \text { in } \bar{D} \times[0, T) .
$$

With (11), we know

$$
\frac{b^{\prime}(u)}{e^{u}} u_{t} \leq \alpha
$$

For each fixed $x \in \bar{D}$, we integrate (32) from 0 to $t$ :

$$
\int_{0}^{t} \frac{b^{\prime}(u)}{e^{u}} u_{t} d t=\int_{u_{0}(x)}^{u(x, t)} \frac{b^{\prime}(s)}{e^{s}} d s \leq \alpha t,
$$

which implies that $u$ must be a global solution of (1). In fact, suppose that $u$ blows up at finite time $T$; then

$$
\lim _{t \rightarrow T^{-}} u(x, t)=+\infty
$$

Passing to the limit as $t \rightarrow T^{-}$in (33) yields

$$
\begin{gathered}
\int_{u_{0}(x)}^{+\infty} \frac{b^{\prime}(s)}{e^{s}} d s \leq \alpha T, \\
\int_{m_{0}}^{+\infty} \frac{b^{\prime}(s)}{e^{s}} d s=\int_{m_{0}}^{u_{0}(x)} \frac{b^{\prime}(s)}{e^{s}} d s+\int_{u_{0}(x)}^{+\infty} \frac{b^{\prime}(s)}{e^{s}} d s \\
\leq \int_{m_{0}}^{u_{0}(x)} \frac{b^{\prime}(s)}{e^{s}} d s+\alpha T<+\infty,
\end{gathered}
$$

which contradicts with the condition (iii). This shows that $u$ is global solution. Moreover, it follows from (33) that

$$
\begin{aligned}
\int_{u_{0}(x)}^{u(x, t)} \frac{b^{\prime}(s)}{e^{s}} d s & =\int_{m_{0}}^{u(x, t)} \frac{b^{\prime}(s)}{e^{s}} d s-\int_{m_{0}}^{u_{0}(x)} \frac{b^{\prime}(s)}{e^{s}} d s \\
& =H(u(x, t))-H\left(u_{0}(x)\right) \leq \alpha t .
\end{aligned}
$$

Since $H$ is an increasing function, we have

$$
u(x, t) \leq H^{-1}\left(\alpha t+H\left(u_{0}(x)\right)\right) .
$$

The proof is completed. 


\section{Blow-Up Solution}

The following theorem is the main result for the blow-up solution of (1).

Theorem 2. Let u be a solution of problem (1). Assume that the following conditions (i)-(iv) are satisfied.

(i) For any $s \in \mathbb{R}^{+}$,

$$
\begin{aligned}
& \left(s b^{\prime}(s)\right)^{\prime} \geq 0, \quad s b^{\prime}(s)-\left(s b^{\prime}(s)\right)^{\prime} \geq 0, \quad\left(\frac{a(s)}{b^{\prime}(s)}\right)^{\prime} \geq 0, \\
& {\left[\frac{1}{a(s)}\left(\frac{a(s)}{b^{\prime}(s)}\right)^{\prime}+\frac{1}{b^{\prime}(s)}\right]^{\prime}+\frac{1}{a(s)}\left(\frac{a(s)}{b^{\prime}(s)}\right)^{\prime}+\frac{1}{b^{\prime}(s)} \geq 0 .}
\end{aligned}
$$

(ii) For any $(x, s, d, t) \in D \times \mathbb{R}^{+} \times \overline{\mathbb{R}^{+}} \times \mathbb{R}^{+}$,

$$
\begin{gathered}
\left(\frac{f(x, s, d, t)}{h(t)}\right)_{t} \geq 0, \\
f_{d}(x, s, d, t)\left[\left(\frac{1}{b^{\prime}(s)}\right)^{\prime}+\frac{1}{b^{\prime}(s)}\right] \geq 0, \\
\left(\frac{f(x, s, d, t) b^{\prime}(s)}{a(s)}\right)_{s}-\frac{f(x, s, d, t) b^{\prime}(s)}{a(s)} \\
+\frac{h^{\prime}(t)\left(b^{\prime}(s)\right)^{2}}{a(s) h(t)} \geq 0 .
\end{gathered}
$$

(iii) Consider the integration

$$
\int_{M_{0}}^{+\infty} \frac{b^{\prime}(s)}{e^{s}} d s<+\infty, \quad M_{0}=\max _{\bar{D}} u_{0}(x) .
$$

(iv) Consider

$$
\begin{array}{r}
\beta=\min _{\bar{D}}\left\{\frac{\nabla \cdot\left(h(0) k(x) a\left(u_{0}\right) \nabla u_{0}\right)+f\left(x, u_{0}, q_{0}, 0\right)}{e^{u_{0}}}\right\}>0, \\
q_{0}=\left|\nabla u_{0}\right|^{2} .
\end{array}
$$

Then the solution $u$ of problem (1) must blow up in finite time $T$, and

$$
\begin{gathered}
T \leq \frac{1}{\beta} \int_{M_{0}}^{+\infty} \frac{b^{\prime}(s)}{e^{s}} d s, \\
u(x, t) \leq G^{-1}(\beta(T-t)), \quad(x, t) \in \bar{D} \times[0, T),
\end{gathered}
$$

where

$$
G(z)=\int_{z}^{+\infty} \frac{b^{\prime}(s)}{e^{s}} d s, \quad z>0,
$$

and $G^{-1}$ is the inverse function of $G$.
Proof. Construct the following auxiliary function:

$$
Q(x, t)=b^{\prime}(u) u_{t}-\beta e^{u} .
$$

So we have

$$
\begin{gathered}
\nabla Q=b^{\prime \prime} u_{t} \nabla u+b^{\prime} \nabla u_{t}-\beta e^{u} \nabla u, \\
\Delta Q=b^{\prime \prime \prime} u_{t}|\nabla u|^{2}+2 b^{\prime \prime} \nabla u \cdot \nabla u_{t}+b^{\prime \prime} u_{t} \Delta u+b^{\prime} \Delta u_{t} \\
-\beta e^{u}|\nabla u|^{2}-\beta e^{u} \Delta u .
\end{gathered}
$$

As the previous derivation from (14) to (25), we can obtain $\frac{a k h}{b^{\prime}} \Delta Q$

$$
+\left[\left(2 k h\left(\frac{a}{b^{\prime}}\right)^{\prime}+\frac{2 f_{q}}{b^{\prime}}\right) \nabla u+\frac{a h}{b^{\prime}} \nabla k\right] \cdot \nabla Q
$$$$
+\left\{\frac{a}{\left(b^{\prime}\right)^{2}}\left(\frac{f b^{\prime}}{a}\right)_{u}+\frac{h^{\prime}}{h}\right.
$$$$
\left.+\left[a k h\left(\frac{1}{a}\left(\frac{a}{b^{\prime}}\right)^{\prime}\right)^{\prime}+2 f_{q}\left(\frac{1}{b^{\prime}}\right)^{\prime}\right]|\nabla u|^{2}\right\} Q-Q_{t}
$$$$
=-\beta e^{u}\left\{2 f_{q}\left[\left(\frac{1}{b^{\prime}}\right)^{\prime}+\frac{1}{b^{\prime}}\right]\right.
$$$$
\left.+a k h\left[\left(\frac{1}{a}\left(\frac{a}{b^{\prime}}\right)^{\prime}+\frac{1}{b^{\prime}}\right)^{\prime}+\frac{1}{a}\left(\frac{a}{b^{\prime}}\right)^{\prime}+\frac{1}{b^{\prime}}\right]\right\}|\nabla u|^{2}
$$$$
-\frac{\left(b^{\prime}\right)^{2}}{a}\left(\frac{a}{b^{\prime}}\right)^{\prime} u_{t}^{2}-\beta e^{u} \frac{a}{\left(b^{\prime}\right)^{2}}
$$$$
\times\left[\left(\frac{f b^{\prime}}{a}\right)_{u}-\frac{f b^{\prime}}{a}+\frac{h^{\prime}\left(b^{\prime}\right)^{2}}{a h}\right]-h\left(\frac{f}{h}\right)_{t}
$$

It is seen from (38) and (39) that the right-hand side of (46) is nonpositive; that is,

$\frac{a k h}{b^{\prime}} \Delta Q$

$$
\begin{aligned}
& +\left[\left(2 k h\left(\frac{a}{b^{\prime}}\right)^{\prime}+\frac{2 f_{q}}{b^{\prime}}\right) \nabla u+\frac{a h}{b^{\prime}} \nabla k\right] \cdot \nabla Q \\
& +\left\{\frac{a}{\left(b^{\prime}\right)^{2}}\left(\frac{f b^{\prime}}{a}\right)_{u}+\frac{h^{\prime}}{h}\right. \\
& \left.+\left[a k h\left(\frac{1}{a}\left(\frac{a}{b^{\prime}}\right)^{\prime}\right)^{\prime}+2 f_{q}\left(\frac{1}{b^{\prime}}\right)^{\prime}\right]|\nabla u|^{2}\right\} Q-Q_{t}
\end{aligned}
$$

$\leq 0, \quad$ in $D \times(0, T)$.

By applying maximum principle (see [17]), it follows from (47) that $Q$ can attain its nonpositive minimum only for $\bar{D} \times\{0\}$ or $\partial D \times(0, T)$. 
For $\bar{D} \times\{0\}$, with (41), we have

$$
\begin{aligned}
& \min _{\bar{D}} Q(x, 0) \\
& =\min _{\bar{D}}\left\{b^{\prime}\left(u_{0}\right)\left(u_{0}\right)_{t}-\beta e^{u_{0}}\right\} \\
& =\min _{\bar{D}}\left\{\nabla \cdot\left(h(0) k(x) a\left(u_{0}\right) \nabla u_{0}\right)\right. \\
& \left.\quad+f\left(x, u_{0}, q_{0}, 0\right)-\beta e^{u_{0}}\right\} \\
& =\min _{\bar{D}}\left\{e ^ { u _ { 0 } } \left[\frac{\nabla \cdot\left(h(0) k(x) a\left(u_{0}\right) \nabla u_{0}\right)+f\left(x, u_{0}, q_{0}, 0\right)}{e^{u_{0}}}\right.\right. \\
& -\beta]\}=0 .
\end{aligned}
$$

For $\partial D \times(0, T)$, substituting $P$ and $\alpha$ with $Q$ and $\beta$ in (29), respectively, we have

$$
\frac{\partial Q}{\partial n}=-\gamma \frac{\left(u b^{\prime}\right)^{\prime}}{b^{\prime}} Q+\gamma \beta e^{u} \frac{u b^{\prime}-\left(u b^{\prime}\right)^{\prime}}{b^{\prime}}, \quad \text { on } \partial D \times(0, T) \text {. }
$$

Combining (47)-(49) with condition (i), we can apply the maximum principles again to obtain that the minimum of $Q$ in $\bar{D} \times[0, T)$ is zero. Thus,

$$
\begin{gathered}
Q \geq 0, \quad \text { in } \bar{D} \times[0, T), \\
\frac{b^{\prime}(u)}{e^{u}} u_{t} \geq \beta .
\end{gathered}
$$

At the point $x^{*} \in \bar{D}$, where $u_{0}\left(x^{*}\right)=M_{0}$, we can integrate (51) from 0 to $t$ to get

$$
\int_{0}^{t} \frac{b^{\prime}(u)}{e^{u}} u_{t} d t=\int_{M_{0}}^{u\left(x^{*}, t\right)} \frac{b^{\prime}(s)}{e^{s}} d s \geq \beta t
$$

which implies that $u$ must blow up in finite time. Actually, if $u$ is a global solution of (1), then, for any $t>0$, (52) shows

$$
\int_{M_{0}}^{+\infty} \frac{b^{\prime}(s)}{e^{s}} d s \geq \int_{M_{0}}^{u\left(x^{*}, t\right)} \frac{b^{\prime}(s)}{e^{s}} d s \geq \beta t
$$

Letting $t \rightarrow+\infty$ in (53), we have

$$
\int_{M_{0}}^{+\infty} \frac{b^{\prime}(s)}{e^{s}} d s=+\infty
$$

which contradicts with assumption (40). This shows that $u$ must blow up in finite time $t=T$. Moreover, letting $t \rightarrow T$ in (52), we have

$$
T \leq \frac{1}{\beta} \int_{M_{0}}^{+\infty} \frac{b^{\prime}(s)}{e^{s}} d s .
$$

By integrating inequality (51) over $[t, s](0<t<s<T)$, for each fixed $x$, we obtain

$$
\begin{aligned}
G(u(x, t)) & \geq G(u(x, t))-G(u(x, s)) \\
& =\int_{u(x, t)}^{+\infty} \frac{b^{\prime}(s)}{e^{s}} d s-\int_{u(x, s)}^{+\infty} \frac{b^{\prime}(s)}{e^{s}} d s \\
& =\int_{u(x, t)}^{u(x, s)} \frac{b^{\prime}(s)}{e^{s}} d s=\int_{t}^{s} \frac{b^{\prime}(u)}{e^{u}} u_{t} d t \geq \beta(s-t) .
\end{aligned}
$$

Hence, by letting $s \rightarrow T$, we have

$$
G(u(x, t)) \geq \beta(T-t) .
$$

Since $G$ is a decreasing function, we obtain

$$
u(x, t) \leq G^{-1}(\beta(T-t)) .
$$

The proof is completed.

\section{Applications}

When $h(t) \equiv 1, k(x) \equiv 1, f(x, u, q, t)=f(u)$ or $b(u)=u$, $h(t) \equiv 1, k(x) \equiv 1, f(x, u, q, t)=f(u)$, or $h(t) \equiv 1$, $k(x) \equiv 1, a(u) \equiv 1, f(x, u, q, t)=f(u)$, the conclusions of Theorems 1 and 2 still hold true. In this sense, our results extend and supplement the results in [14-16]. In what follows, we present several examples to demonstrate the applications of the abstract results.

Example 1. Let $u$ be a solution of the following problem:

$$
\begin{aligned}
&\left(u e^{u}\right)_{t}= \nabla \cdot\left(\frac{1}{1+t} e^{|x|^{2}}(1+u) e^{u} \nabla u\right) \\
&+ \frac{1}{1+t}\left(e^{-u}+e^{q}\right)\left(e^{-t}+|x|^{2}\right), \quad \text { in } D \times(0, T), \\
& \frac{\partial u}{\partial n}+2 u=0, \quad \text { on } \partial D \times(0, T), \\
& u(x, 0)=2-|x|^{2}, \quad \text { in } \bar{D},
\end{aligned}
$$

where $q=|\nabla u|^{2}, D=\left\{x=\left.\left(x_{1}, x_{2}, x_{3}\right)|| x\right|^{2}<1\right\}$ is the unit ball of $\mathbb{R}^{3}$. Now we have

$$
\begin{gathered}
b(u)=u e^{u}, \quad h(t)=\frac{1}{1+t}, \\
k(x)=e^{|x|^{2}}, \quad a(u)=(1+u) e^{u}, \quad \gamma=2, \\
f(x, u, q, t)=\frac{1}{1+t}\left(e^{-u}+e^{q}\right)\left(e^{-t}+|x|^{2}\right), \\
u_{0}(x)=2-|x|^{2} .
\end{gathered}
$$

In order to determine the constant $\alpha$, we assume

$$
s=|x|^{2},
$$


and then $0 \leq s \leq 1$ and

$$
\begin{aligned}
\alpha=\max _{\bar{D}} \frac{\nabla \cdot\left(h(0) k(x) a\left(u_{0}\right) \nabla u_{0}\right)+f\left(x, u_{0}, q_{0}, 0\right)}{e^{u_{0}}} \\
=\max _{\bar{D}}\left\{e^{|x|^{2}}\left(10|x|^{2}-18\right)+\left(1+|x|^{2}\right)\right. \\
\left.\quad \times\left(e^{-4+2|x|^{2}}+e^{-2+5|x|^{2}}\right)\right\} \\
=\max _{0 \leq s \leq 1}\left\{e^{s}(10 s-18)+(1+s)\right. \\
\left.\quad \times\left[e^{-4+2 s}+e^{-2+5 s}\right]\right\}=18.6955 .
\end{aligned}
$$

It is easy to check that (5)-(7) hold. By Theorem 1, $u$ must be a global solution, and

$$
\begin{aligned}
u(x, t) & \leq H^{-1}\left(\alpha t+H\left(u_{0}(x)\right)\right) \\
& =-1+\sqrt[2]{18.6955 t+\left(1+u_{0}(x)\right)^{2}} \\
& =-1+\sqrt[2]{18.6955 t+\left(3-|x|^{2}\right)^{2}} .
\end{aligned}
$$

Example 2. Let $u$ be a solution of the following problem:

$$
\begin{array}{r}
(u+\ln u)_{t}=\nabla \cdot\left((1+t) e^{-|x|^{2}}\left(1+\frac{1}{u}\right) \nabla u\right) \\
+(1+t)\left(e^{u}-e^{-q}\right)\left(6+t|x|^{2}\right), \\
\text { in } D \times(0, T), \\
\frac{\partial u}{\partial n}+2 u=0, \quad \text { on } \partial D \times(0, T), \\
u(x, 0)=2-|x|^{2}, \quad \text { in } \bar{D},
\end{array}
$$

where $q=|\nabla u|^{2}, D=\left\{x=\left.\left(x_{1}, x_{2}, x_{3}\right)|| x\right|^{2}<1\right\}$ is the unit ball of $\mathbb{R}^{3}$. Now we have

$$
\begin{gathered}
b(u)=u+\ln u, \quad h(t)=1+t, \\
k(x)=e^{-|x|^{2}}, \quad a(u)=1+\frac{1}{u}, \quad \gamma=2, \\
f(x, u, q, t)=(1+t)\left(e^{u}-e^{-q}\right)\left(6+t|x|^{2}\right), \\
u_{0}(x)=2-|x|^{2} .
\end{gathered}
$$

In order to determine the constant $\beta$, we assume

$$
s=|x|^{2}
$$

and then $0 \leq s \leq 1$ and

$$
\begin{aligned}
& \beta=\min _{\bar{D}} \frac{\nabla \cdot\left(h(0) k(x) a\left(u_{0}\right) \nabla u_{0}\right)+f\left(x, u_{0}, q_{0}, 0\right)}{e^{u_{0}}} \\
& =\min _{\bar{D}}\left\{\frac{4|x|^{6}-26|x|^{4}+50|x|^{2}-36}{\left(2-|x|^{2}\right)^{2} e^{2}}\right. \\
& \left.+6\left(1-e^{-3|x|^{2}-2}\right)\right\} \\
& =\min _{0 \leq s \leq 1}\left\{\frac{4 s^{3}-26 s^{2}+50 s-36}{(2-s)^{2} e^{2}}\right. \\
& \left.+6\left(1-e^{-3 s-2}\right)\right\}=3.96997 \text {. }
\end{aligned}
$$

It is easy to check that (38)-(40) hold. By Theorem 2, $u$ must blow up in finite time $T$, and

$$
\begin{aligned}
T & \leq \frac{1}{\beta} \int_{M_{0}}^{+\infty} \frac{b^{\prime}(s)}{e^{s}} d s \\
& =\frac{1}{3.96997} \int_{2}^{+\infty}\left(1+\frac{1}{s}\right) \frac{1}{e^{s}} d s=0.04641, \\
u(x, t) & \leq G^{-1}(\beta(T-t))=G^{-1}(3.96997(T-t)),
\end{aligned}
$$

where

$$
G(z)=\int_{z}^{+\infty} \frac{b^{\prime}(s)}{e^{s}} d s=\int_{z}^{+\infty}\left(1+\frac{1}{s}\right) \frac{1}{e^{s}} d s, \quad z \geq 0,
$$

and $G^{-1}$ is the inverse function of $G$.

\section{Conflict of Interests}

The authors declare that there is no conflict of interests regarding the publication of this paper.

\section{Acknowledgments}

The authors especially thank the reviewers for their very useful suggestions. The research was partially supported by the National Natural Science Foundation of China (no. 61250011) and the Science Foundation of Shanxi Province (no. 2012011004-4) and Postdoctoral Science Foundation (no. 2012M510786).

\section{References}

[1] A. Constantin and J. Escher, "Well-posedness, global existence, and blowup phenomena for a periodic quasi-linear hyperbolic equation," Communications on Pure and Applied Mathematics, vol. 51, no. 5, pp. 475-504, 1998.

[2] J. M. Chadam, A. Peirce, and H. Yin, "The blowup property of solutions to some diffusion equations with localized nonlinear reactions," Journal of Mathematical Analysis and Applications, vol. 169, no. 2, pp. 313-328, 1992. 
[3] J. Wang, Z. J. Wang, and J. X. Yin, "A class of degenerate diffusion equations with mixed boundary conditions," Journal of Mathematical Analysis and Applications, vol. 298, no. 2, pp. 589-603, 2004.

[4] N. Wolanski, "Global behavior of positive solutions to nonlinear diffusion problems with nonlinear absorption through the boundary," SIAM Journal on Mathematical Analysis, vol. 24, no. 2, pp. 317-326, 1993.

[5] J. Ding, "Blow-up of solutions for a class of semilinear reaction diffusion equations with mixed boundary conditions," Applied Mathematics Letters, vol. 15, no. 2, pp. 159-162, 2002.

[6] L. Zhang, "Blow-up of solutions for a class of nonlinear parabolic equations," Zeitschrift für Analysis und ihre Anwendungen, vol. 25, no. 4, pp. 479-486, 2006.

[7] L. A. Caffarelli and A. Friedman, "Blowup of solutions of nonlinear heat equations," Journal of Mathematical Analysis and Applications, vol. 129, no. 2, pp. 409-419, 1988.

[8] V. A. Galaktionov and J. L. Vázquez, "The problem of blowup in nonlinear parabolic equations," Discrete and Continuous Dynamical Systems, vol. 8, no. 2, pp. 399-433, 2002.

[9] H. Zhang and X. Guo, "Blow-up for nonlinear heat equations with absorptions," European Journal of Pure and Applied Mathematics, vol. 1, no. 3, pp. 33-39, 2008.

[10] A. A. Samarskii, V. A. Galaktionov, S. P. Kurdyumov, and A. P. Mikhailov, Blow-Up in Problems for Quasilinear Parabolic Equations, Nauka, Moscow, Russia, 1987, (Russian), English Translation: Walter de Gruyter, Berlin, Germany, 1995.

[11] L. E. Payne and P. W. Schaefer, "Lower bounds for blow-up time in parabolic problems under Neumann conditions," Applicable Analysis, vol. 85, no. 10, pp. 1301-1311, 2006.

[12] H. Amann, "Quasilinear parabolic systems under nonlinear boundary conditions," Archive for Rational Mechanics and Analysis, vol. 92, no. 2, pp. 153-192, 1986.

[13] J. Ding and S. Li, "Blow-up solutions for a class of nonlinear parabolic equations with mixed boundary conditions," Journal of Systems Science and Complexity, vol. 18, no. 2, pp. 265-276, 2005.

[14] J. Ding, "Global and blow-up solutions for nonlinear parabolic equations with Robin boundary conditions," Computers \& Mathematics with Applications, vol. 65, no. 11, pp. 1808-1822, 2013.

[15] C. Enache, "Blow-up phenomena for a class of quasilinear parabolic problems under Robin boundary condition," Applied Mathematics Letters, vol. 24, no. 3, pp. 288-292, 2011.

[16] H. Zhang, "Blow-up solutions and global solutions for nonlinear parabolic problems," Nonlinear Analysis: Theory, Methods \& Applications, vol. 69, no. 12, pp. 4567-4574, 2008.

[17] R. P. Sperb, Maximum Principles and Their Applications, vol. 157 of Mathematics in Science and Engineering, Academic Press, New York, NY, USA, 1981. 


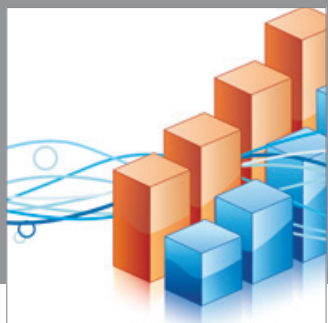

Advances in

Operations Research

mansans

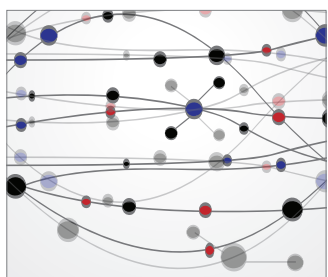

The Scientific World Journal
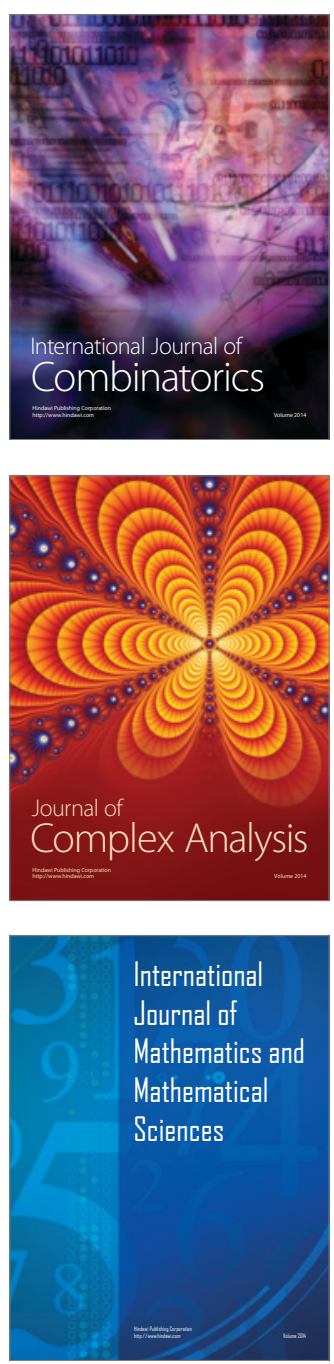
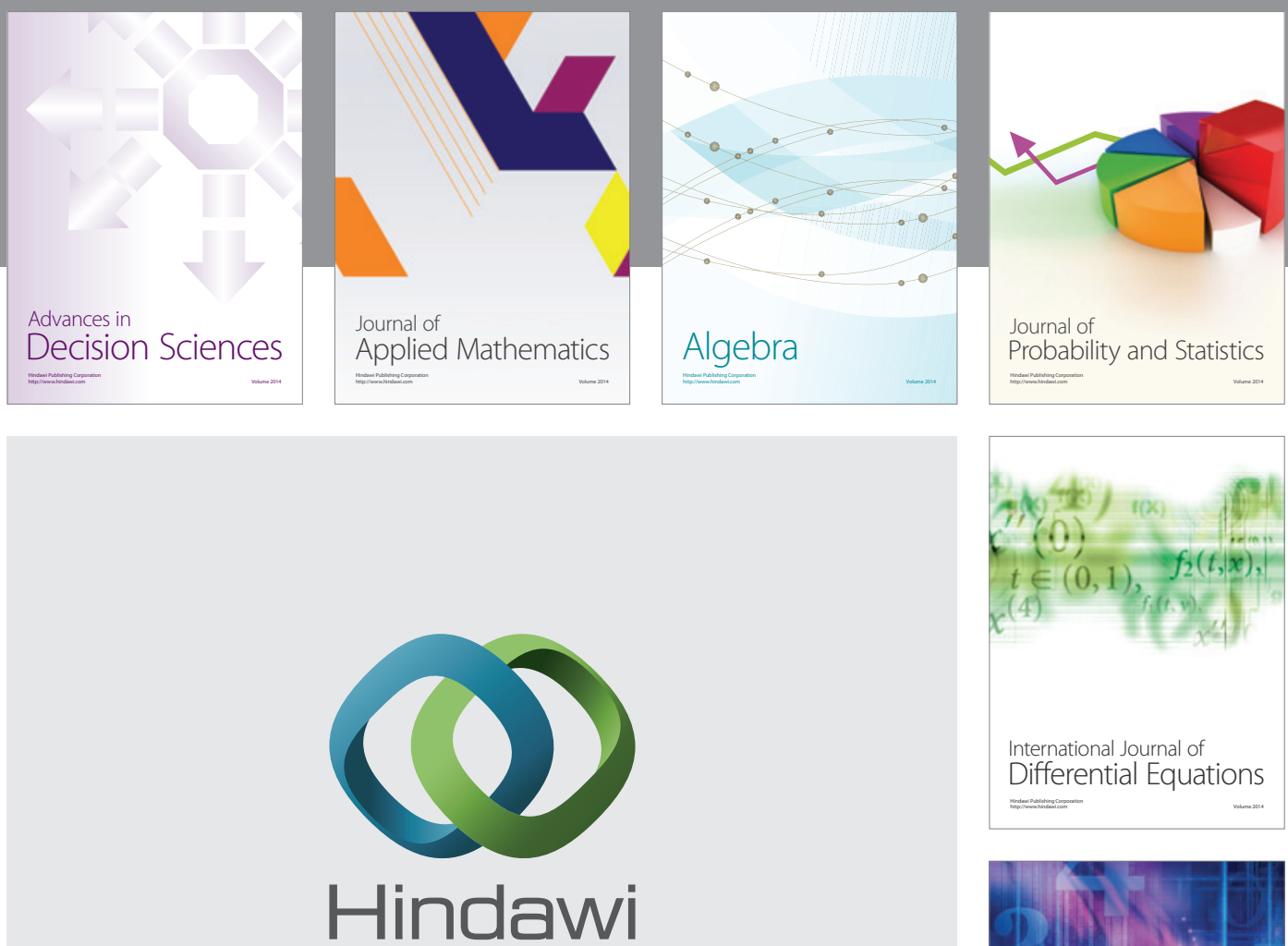

Submit your manuscripts at http://www.hindawi.com
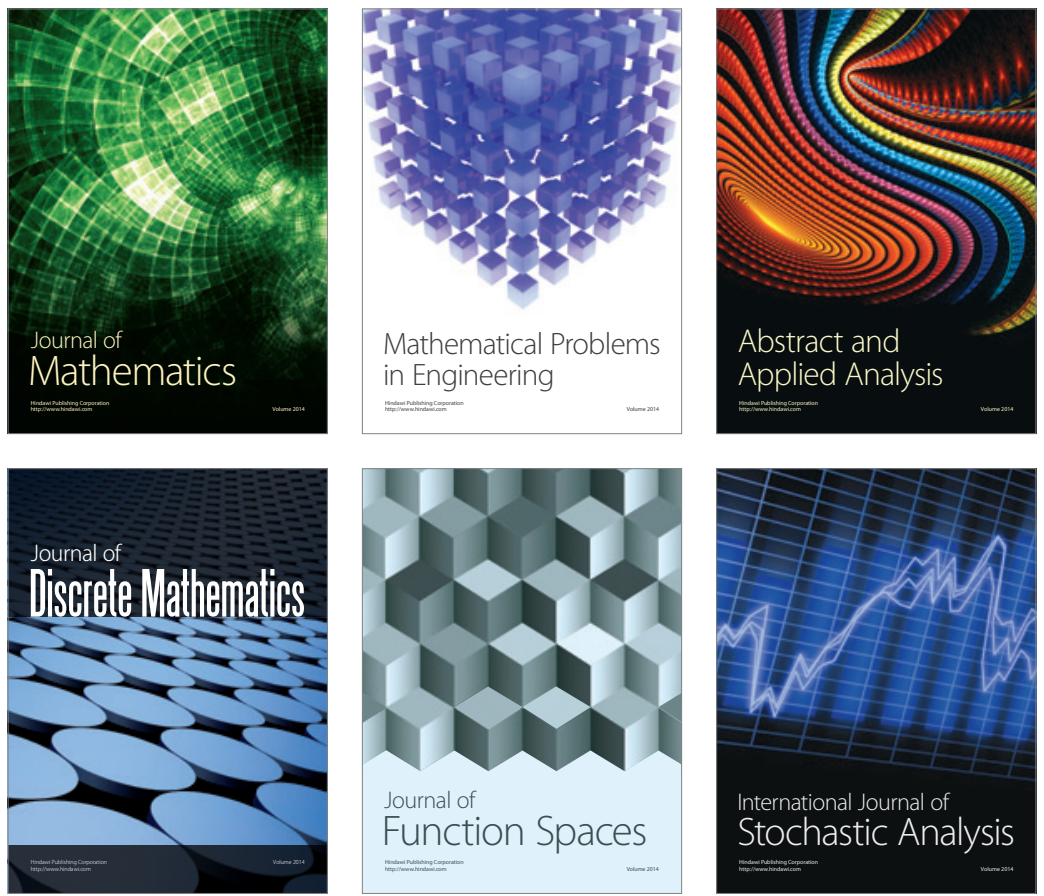

Journal of

Function Spaces

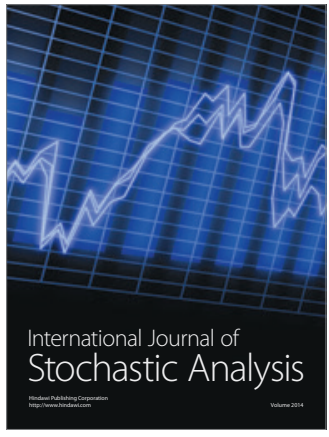

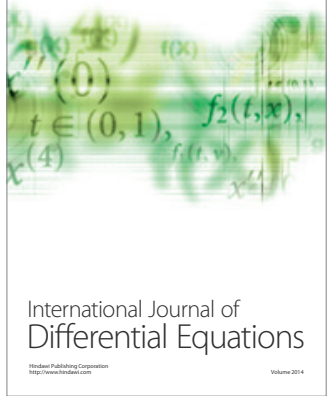
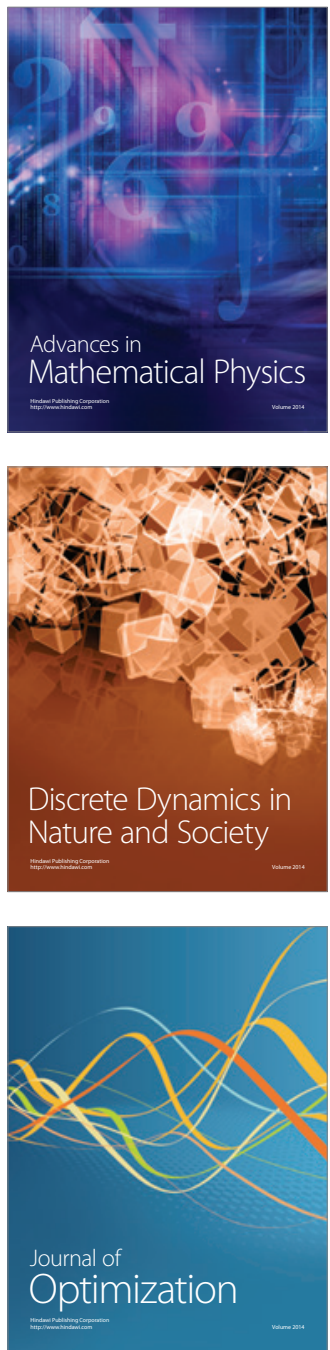